\title{
JNK1, but Not JNK2, Is Required in Two Mechanistically Distinct Models of Inflammatory Arthritis
}

\author{
Katja Denninger, ${ }^{*}$ Susanne Rasmussen, ${ }^{\dagger}$ \\ Jeppe Madura Larsen, ${ }^{\dagger}$ Catrine Ørskov, ${ }^{\ddagger}$ \\ Steen Seier Poulsen, ${ }^{\ddagger}$ Poul Sørensen, ${ }^{\S}$ \\ Jan Pravsgaard Christensen, ${ }^{\dagger}$ Harald IIIges, ${ }^{\natural}$ \\ Niels Ødum, ${ }^{\dagger \mid}$ and Tord Labuda ${ }^{\ddagger \S}$

\begin{abstract}
From the Departments of Pharmacology and Pharmacotherapy," International Health, Immunology and Microbiology, ${ }^{\dagger}$ Medical Anatomy, ${ }^{\ddagger}$ and Molecular Biology and Physiology," Institute of Molecular Biology, University of Copenhagen, Copenhagen, Denmark; Biological Research, ${ }^{\varsigma}$ LEO Pharma A/S, Ballerup, Denmark; and Department of Natural Sciences, "Immunology and Cell Biology, University of Applied Sciences Bonn-Rhein-Sieg, Rheinbach, Germany
\end{abstract}

The roles of the c-Jun N-terminal kinases (JNKs) in inflammatory arthritis have been investigated; however, the roles of each isotype (ie, JNK1 and JNK2) in rheumatoid arthritis and conclusions about whether inhibition of one or both is necessary for amelioration of disease are unclear. By using JNK1- or JNK2-deficient mice in the collagen-induced arthritis and the KRN T-cell receptor transgenic mouse on $\mathrm{C} 57 \mathrm{BL} / 6$ nonobese diabetic (K/ BxN) serum transfer arthritis models, we demonstrate that JNK1 deficiency results in protection from arthritis, as judged by clinical score and histological evaluation in both models of inflammatory arthritis. In contrast, abrogation of JNK 2 exacerbates disease. In collagen-induced arthritis, the distinct roles of the JNK isotypes can, at least in part, be explained by altered regulation of CD86 expression in JNK1- or JNK2-deficient macrophages in response to microbial products, thereby affecting T-cell-mediated immunity. The protection from $\mathrm{K} / \mathrm{BxN}$ serum-induced arthritis in $J n k 1^{-1-}$ mice can also be explained by inept macrophage function because adoptive transfer of wild-type macrophages to $J n k 1^{-/-}$mice restored disease susceptibility. Thus, our results provide a possible explanation for the modest therapeutic effects of broad JNK inhibitors and suggest that future therapies should selectively target the JNK1 isoform. (Am J Patbol 2011, 179:1884-1893; DOI: 10.1016/j.ajpath.2011.06.019)
Rheumatoid arthritis (RA) is a chronic autoimmune disease that affects multiple joints. RA is characterized by infiltration of immune cells, inflammation, pannus formation, and joint destruction. It is generally accepted that pro-inflammatory cytokines play an important role in the pathogenesis of RA. ${ }^{1}$ However, despite intense investigation, the cause of RA remains unknown.

The mitogen-activated protein kinases known as the c-Jun N-terminal kinases (JNKs) participate in RA, ${ }^{2}$ and their role has been investigated in several in vivo studies. $^{3-5}$ The inhibition of JNK by using SP600125, which prevents both JNK1 and JNK2 activity, in a rat model of adjuvant arthritis resulted in a modest decrease in paw swelling and inflammation but showed a significant inhibition of radiographical damage. ${ }^{3}$ The influence of JNK2 was investigated in passive collagen-induced arthritis (CIA) using JNK2-deficient mice. ${ }^{4}$ In this study, the severity of arthritis was slightly increased in the JNK2-deficient animals, whereas the degree of synovial inflammation was comparable to that in wild-type (wt) mice. The influence of JNK1 was investigated in yet another model of autoimmune inflammation, tumor necrosis factor (TNF)- $\alpha$-mediated inflammatory joint disease. ${ }^{5}$ Results from this study suggested that JNK1 is not required for TNF- $\alpha$-mediated arthritis. However, none of these studies has com-

Supported by grants from The Danish Medical Research Councils (S.S.F.), The Leo Research Foundation, The Rheumatism Society (Gigtforeningen), Denmark, The Becket Foundation, The Lundbeck Foundation, The Novo Nordisk Research Foundation, and The University of Copenhagen.

Accepted for publication June 20, 2011.

K.D. and S.R. contributed equally to this work.

Supplemental material for this article can be found at $h$ ttp://ajp. amjpathol.org or at doi:10.1016/j.ajpath.2011.06.019.

Current address of J.M.L., Department of Systems Biology, Center for Biological Sequence Analysis, Technical University of Denmark, Kongens Lyngby, Denmark; of C.Ø., Novo Nordisk A/S, Maaloev, Denmark; of P.S., ALK ABELLÓ, Hoersholm, Denmark.

Address reprint requests to Tord Labuda, M.Sc., Ph.D., Biological Research, LEO Pharma A/S, Industriparken 55, Ballerup 2750, Denmark. E-mail: tord.labuda@leo-pharma.com. 
pared JNK1- with JNK2-deficient animals in the same model of inflammatory arthritis. Thus, the conclusion from these studies about whether selective inhibition of one isoform or nonselective inhibition of JNK1 and JNK2 is required for major blockade of inflammatory arthritis calls for speculation. Recently, however, Guma and coworkers ${ }^{6,7}$ have suggested that JNK1, but not JNK2, is critical for the induction of antibody-induced arthritis (AIA) and serum-induced arthritis; they speculate that this is due to diminished macrophage migration in the AIA model and mast cell degranulation and cytokine production in the serum-induced arthritis model.

In the present study, we chose two well-defined mouse models of inflammatory arthritis, CIA and the KRN T-cell receptor transgenic mouse on C57BL/6 $\times$ nonobese diabetic $(\mathrm{K} / \mathrm{B} \times \mathrm{N})$ serum transfer model, to directly compare the importance of JNK1 with JNK2 during the autoimmune initiation phase and the T-cellindependent effector phase of experimental arthritis, respectively.

$\mathrm{CIA}$ is an autoimmune disease of the joints requiring both $\mathrm{T}$ - and B-cell immunity to type II collagen and macrophages for disease manifestation. ${ }^{8}$ Although $T$ cells play a prominent role in classic CIA, the primary mechanism for the immunopathogenesis seems to be autoantibodies to type II collagen that subsequently bind to the joint cartilage and activate the complement system. ${ }^{9}$ This model was originally described for genetically susceptible strains bearing the major histocompatibility complex (MHC) haplotypes $\mathrm{H}-2^{a}$ or $\mathrm{H}-2^{r}$, respectively (ie, DBA/1, B10.Q and B10.RIII). Recently, an altered protocol for the development of $\mathrm{CIA}$ in $\mathrm{H}-2^{\mathrm{b}}-$ bearing C57BL/6 mice was described. ${ }^{10}$ This disease resembles classic CIA both clinically and histologically and requires $\mathrm{CD} 4^{+} \mathrm{T}$ and $\mathrm{B}$ cells for development. Interestingly, $\mathrm{CIA}$ in $\mathrm{B} 6$ mice is milder but more chronic, with a more pronounced and persistent T-cell response, compared with classic CIA in DBA/1 mice. ${ }^{11}$ The K/BxN model of serum-induced arthritis is independent of both $\mathrm{B}$ and $\mathrm{T}$ cells, external antigen, and adjuvant for disease development. ${ }^{12}$ Thus, this serum transfer system allows us to focus on the inflammatory effector phase of disease, without the complicating influences of the autoimmune initiation phase. By using these models, we provide evidence showing that JNK1 is required for the initiation and effector phases of inflammatory arthritis, whereas deficiency in JNK2 exacerbates the clinical signs of the inflammatory response.

\section{Materials and Methods}

\section{Mice}

All mice were bred and maintained in the animal facility of the Panum Institute, University of Copenhagen, Copenhagen, Denmark. Jnk1 $1^{-1-}$ and Jnk2 $2^{-1-}$ mice were previously described ${ }^{13,14}$ and were backcrossed to C57BL/6 mice for at least eight generations. KRN T-cell receptor transgenic mice have also been described. ${ }^{15}$ These studies have been reviewed and approved by the local ethical committee.

\section{ClA Model}

The initiation of CIA in C57BL/6 mice has been previously described. ${ }^{10}$ Briefly, Complete Freund's Adjuvant (CFA) was prepared in our laboratory by dissolving $100 \mathrm{mg}$ of heat-killed tuberculosis (H37Ra; Difco Laboratories, Detroit, MI) in $20 \mathrm{~mL}$ IFA (incomplete Freund's adjuvant) (Sigma, St Louis, MO). A $2 \mathrm{mg} / \mathrm{mL}$ chick type II collagen preparation (Sigma) was dissolved in $10 \mathrm{mmol} / \mathrm{L}$ acetic acid by gentle agitation overnight at $4^{\circ} \mathrm{C}$ and then emulsified with an equal volume of CFA. Mice were injected intradermally into the base of the tail with a total volume of 100- $\mu \mathrm{L}$ emulsion containing $100 \mu \mathrm{g}$ collagen type II (CII) and $250 \mu \mathrm{g}$ of Mycobacterium tuberculosis. Booster immunizations were given 21 days later. To quantify the intensity of CIA, each animal was assessed for redness and swelling of limbs and assigned a clinical score three times per week. The score was composed of the sum of involvement of each limb. The scale for each limb was as follows: 0 , no clinical symptoms; 1 , slight swelling; 2 , extensive swelling; and 3, joint distortion and/or rigidity. The maximum score per mouse was 12 .

\section{Histological Assessment of CIA}

When sacrificed, the limbs of the mice were removed, fixed in 10\% formalin, and decalcified in EDTA. Paraffinembedded sections were stained with Mayer's H\&E. ${ }^{16}$ The severity of arthritis was blindly evaluated using three parameters: infiltration of monomorphonuclear and polymorphonuclear cells, hyperplasia of the synovium, and bone destruction. Each parameter was scored on a scale from 0 to 3 , as follows: 0 , absent; 1 , weak; 2 , moderate; and 3 , severe.

\section{Serum-Induced Arthritis}

Serum samples were collected from K/BxN mice, aged 1 to 3 months, and pooled. wt, Jnk $1^{-1-}$, and Jnk2 ${ }^{-1-}$ male mice were injected i.p. with $200 \mu \mathrm{L}$ of serum on days 0 and 2. ${ }^{17}$ The development of arthritis was assessed by caliper (Mitotoyo, Tokyo, Japan), with ankle thickening defined as the difference in the ankle thickness from the day 0 measure. A clinical index was evaluated for each paw, according to the following criteria: 0 , normal; 0.5, mild swelling of the paws or a few digits affected; and 1.0, clear inflammation in the ankle or joint. The score of the mice was evaluated blindly.

\section{Generation of BMMs}

The bone marrow macrophages (BMMs) obtained from wt Jnk $1^{-1-}$ and Jnk $2^{-1-}$ mice were cultured for 6 days in Dulbecco's modified Eagle's medium (Sigma), supplemented with $25 \%(\mathrm{v} / \mathrm{v})$ fetal bovine serum and $25 \%(\mathrm{v} / \mathrm{v})$ L929 cell-conditioned media. The cells were synchronized by culture with $10 \%$ fetal bovine serum for 24 hours. 


\section{Adoptive Transfer of BMMs}

The BMMs were isolated from wt donor mice and differentiated as previously described. On days $-1,2$, and 4 , $1 \times 10^{6}$ macrophages were injected into the tail vein of Jnk $1^{-1-}$ recipient mice. wt, Jnk $1^{-1-}$, and Jnk $1^{-1-}$ BMM recipient male mice were injected i.p. with $200 \mu \mathrm{L}$ of serum on days 0 and 2 .

\section{Enzyme-Linked Immunosorbent Assay for Cll-Specific Antibodies}

Serum was isolated from individual animals and stored at $-80^{\circ} \mathrm{C}$. The concentration of total anti-Cll-specific IgG was determined according to the manufacturer's instructions (MD Biosciences $\mathrm{GmbH}$, Zürich, Switzerland). For Cll-specific IgG1 and IgG2a determinations, $2 \mu \mathrm{g} / \mathrm{mL}$ of $\mathrm{Cll}$ was diluted in Tris- $\mathrm{NaCl}(\mathrm{pH} \mathrm{7.4)}$ and used for overnight coating of enzyme-linked immunosorbent assay plates (Nunc, Roskilde, Denmark). Serum samples were diluted 1:10,000 and incubated for 1 hour at room temperature. After washing, the plates were incubated for 1 hour at room temperature with horseradish peroxidaseconjugated IgG1 (Bethyl Laboratories, Inc., Montgomery, TX), diluted 1:50,000, or horseradish peroxidase-conjugated goat anti-mouse IgG2a (Bethyl Laboratories, Inc.), diluted 1:30,000. The reaction was developed with TMB for 1 hour and discontinued with $\mathrm{H}_{2} \mathrm{SO}_{4}$. The absorbance was measured at $450 \mathrm{~nm}$ in an enzyme-linked immunosorbent assay reader.

\section{Quantitative PCR}

Total RNA was isolated from macrophages as previously described. ${ }^{18}$ Quantitative RT-PCR analysis was performed using the SYBR Green PCR Master Mix kit, according to the manufacturer's recommendations (Stratagene, AH Diagnostics, Aarhus, Denmark) using the following primers: TNF- $\alpha, 5$ '-GCACAGAAAGCATGACCCG-3' (forward) and 5'-GCCCCCCATCTTTTGGG-3' (reverse) (annealing temperature, $58^{\circ} \mathrm{C}$ ); IL-1 $\beta$, 5'CAACCAACAAGTGATATTCTCCATG-3' (forward) and 5'GATCCACACTCTCCAGCTGCA-3' (reverse) (annealing temperature, $61^{\circ} \mathrm{C}$ ); and IL-10, $5^{\prime}$-GGTTGCCAAGCCTTATCGGA-3' (forward) and 5'-ACCTGCTCCACTGCCTTGCT-3' (reverse) (annealing temperature, $60^{\circ} \mathrm{C}$ ). All primers were from DNA Technology (Risskov, Denmark). The PCR was initiated with $95^{\circ} \mathrm{C}$ for 10 minutes, and the PCR cycling conditions were as follows: $95^{\circ} \mathrm{C}$ for 30 seconds, the previously mentioned specific annealing temperature for 1 minute, and $72^{\circ} \mathrm{C}$ for 30 seconds for 40 cycles. The quantitative PCR values were normalized using a specific cDNA standard curve obtained using known amounts of cDNA. TNF- $\alpha, I L-1 \beta$, and IL-10 were then normalized to glyceraldehyde-3-phophate dehydrogenase.

\section{Flow Cytometry}

Cells were stained with fluorochrome-conjugated primary antibodies (Abs) for 30 minutes at $4^{\circ} \mathrm{C}$ in PBS containing $0.5 \%$ bovine serum albumin, washed twice, acquired on an FACSCalibur flow cytometer (BD Biosciences, Broendby, Denmark) and analyzed with FlowJo Software (Tree Star, OR), or acquired on a BD LSR II flow cytometer (BD Biosciences) and analyzed with BD FACSDiva software. Abs against $F 4 / 80, C D 86$, and $1-E^{k}$ were obtained from Pharmingen (Leiden, The Netherlands), whereas Abs against CD4 and 7-aminoactinomycin D (7-AAD) were obtained from BD Biosciences.

\section{Western Blot Analysis}

Whole cell lysate was resolved by SDS-PAGE and electroblotted onto nitrocellulose membranes. The membranes were incubated with primary antibodies in 5\% nonfat milk overnight at $4^{\circ} \mathrm{C}$ and secondary antibodies for 1 hour at room temperature. The electrochemiluminescence detection method was used for all Western blot experiments.

\section{DTH Model}

wt, Jnk $1^{-1-}$, and Jnk2 $2^{-1-}$ mice were sensitized on day 0 , as described for CIA. On day 17, delayed-type hypersensitivity (DTH) responses were induced intradermally in the right ear by injection of $10 \mu \mathrm{g} \mathrm{Cll}$ in $2.5 \mathrm{mmol} / \mathrm{L}$ acetic acid. Vehicle, $20 \mu \mathrm{L}$, was injected in the left ear as a control. Ear thickness was measured using a micrometer before and 18 , 24 , and 48 hours after challenge with antigen.

\section{Cutaneous Contact Hypersensitivity Model}

On days 0 and 1 , shaved dorsal skins of mice were painted with $0.5 \%$ 2,4-dinitrofluorobenzene (DNFB) in $25-\mu \mathrm{L}$ acetone. On day 5 , the right ear was challenged with $0.25 \%$ DNFB and the left ear was painted with vehicle (acetone). Ear thickness to the first cartilage ridge was measured 24 hours later. To account for acute hapten-induced irritation, background swelling was measured in parallel with that of nonsensitized mice. Specific ear swelling was calculated as follows: (treated ear thickness - control ear thickness) - background swelling.

\section{T-Cell Proliferation Assay}

The RAW 264.7 murine macrophage cell line from American Type Culture Collection (Manassas, VA) was cultured in Dulbecco's modified Eagle's medium (Sigma) for 24 hours before treatment with $25 \mu \mathrm{mol} / \mathrm{L}$ SP600125 JNK inhibitor (Sigma) (concentration determined in preceding trials) or dimethylsulfoxide (control) for 2 hours before stimulation with lipopolysaccharide (LPS; $1 \mu \mathrm{g} / \mathrm{mL}$ ) for 24 hours. After LPS stimulation, the cells were treated for 30 minutes at $37^{\circ} \mathrm{C}$ with mitomycin $\mathrm{C}$ (Sigma), washed 5 times in ice-cold PBS, and cultured in a 96-well plate with 30,000 cells/well in RPMI 1640 medium supplemented with 10\% fetal bovine serum, 1\% GlutaMax (Invitrogen, Hellerup, Denmark), and 1\% penicillin-streptomycin.

Primary $T$ cells were isolated from the spleen of 6 - to 8-week-old C57BL/6JBom mice using negative selection (EasySep Mouse T Cell Enrichment Kit; StemCell Technologies, Grenoble, France), according to the 

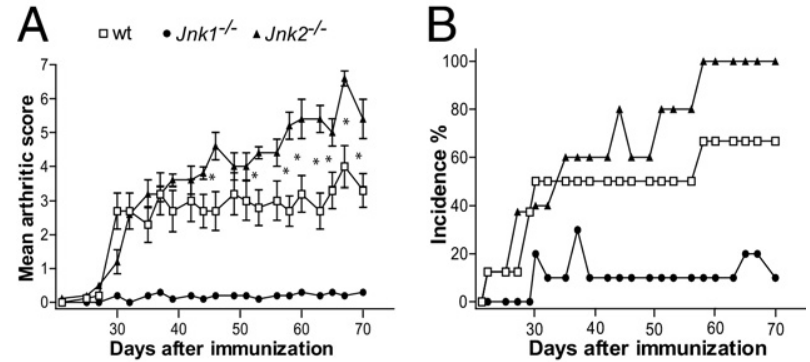

Figure 1. $J n k 1^{-/-}$mice are resistant to CIA, whereas $J n k 2^{-/-}$mice are highly susceptible. A: Clinical arthritis scores. B: Cumulative incidence of arthritis in wt, $J n k 1^{-/-}$, and $J n k 2^{-/-}$mice with CIA. The severity of arthritis was evaluated using a scoring system, as described in Materials and Methods. Results are presented as the mean $\pm \mathrm{SD} .{ }^{*} P<0.05\left(n=20\right.$ for $J n k 1^{-/-}$, $n=10$ for $J n k 2^{-/-}$, and $n=15$ for $\mathrm{wt}$ ).

manufacturer's protocol. The T cells were then labeled with $1 \mu \mathrm{mol} / \mathrm{L}$ carboxyfluorescein diacetate succinimyl ester (Molecular Probes; Invitrogen) for 10 minutes at $37^{\circ} \mathrm{C}$, resuspended in $10 \times$ ice-cold $\mathrm{PBS} / 10 \%$ fetal bovine serum, and incubated on ice for 5 minutes. Next, 100,000 T cells/well were co-cultured with mitomycin C-treated RAW 264.7 cells, supplemented with 0.3 $\mu \mathrm{g} / \mathrm{mL}$ anti-mouse CD3e Abs (eBioscience, San Diego, $\mathrm{CA}$ ) and $10 \mathrm{ng} / \mathrm{mL}$ murine recombinant IL-2 (R\&D Systems, Minneapolis, MN). Assessment of proliferation was judged after 96 hours using flow cytometry.

\section{Statistics}

Quantitative data are expressed as the mean \pm SEM if nothing else is stated in the figure legend. Significance analysis was performed using the Student's $t$-test or the $U$-test. All results were compared with wt mice with the exception of the BMM reconstitution experiment in which Jnk $1^{-1-}$ mice reconstituted with wt BMMs were compared with Jnk $1^{-1-}$ mice.

\section{Results}

\section{Jnk $1^{-/-}$Mice Are Resistant to CIA}

To examine the role of JNK1 and JNK2 in a model of chronic autoinflammatory arthritis, C57BL/6 mice deficient for either JNK1 or JNK2 were compared with wt mice for incidence and severity of $\mathrm{ClA}$, induced by intradermal injection of CII in CFA, followed by a boost injection 21 days later. Of wt mice, 67\% $(n=15)$ developed arthritis, with a clinical score of $3.3 \pm 1.2$ at termination of the experiment (day 70 after immunization). JNK1-deficient mice $(n=20)$ were almost completely protected from CIA, although 15\% (3/20) of the JNK1-deficient mice developed weak arthritis, with a score of $0.3 \pm 0.5$. In contrast, JNK2deficient mice were highly susceptible to CIA, and all of these mice $(n=10)$ developed severe arthritis, showing a clinical score of $5.4 \pm 1.3$ at day 70 (Figure 1).

\section{Histological Analysis Confirms the Lack of Inflammation in Joints from JNK1-Deficient Mice}

To confirm the clinical analysis, H\&E-stained sections of joints from wt, JNK1- deficient, and JNK2-deficient mice underwent histological examination. Joints from wt and Jnk2 ${ }^{-1-}$ mice showed severe inflammation and tissue destruction, with extensive hyperplasia of the synovium (Figure 2, A and B). However, although the arthritic score observed in Jnk2 $2^{-1-}$ mice was significantly higher compared with wt mice during the last 20 days of the disease course, no difference in histological analysis, including bone destruction, hyperplasia of the synovium, or infiltration of inflammatory cells, was detected between the groups. In contrast, joints from Jnk $1^{-1-}$ mice appeared normal and no (or minimal) tissue destruction and infiltration of inflammatory cells was observed (Figure 2, A and B).

\section{Altered Humoral Immunity in Response to $\mathrm{Cll}$ in JNK1- and JNK2-Deficient Mice}

CIA is dependent on both humoral and cellular immunity. ${ }^{19}$ To establish a possible explanation for the diver-
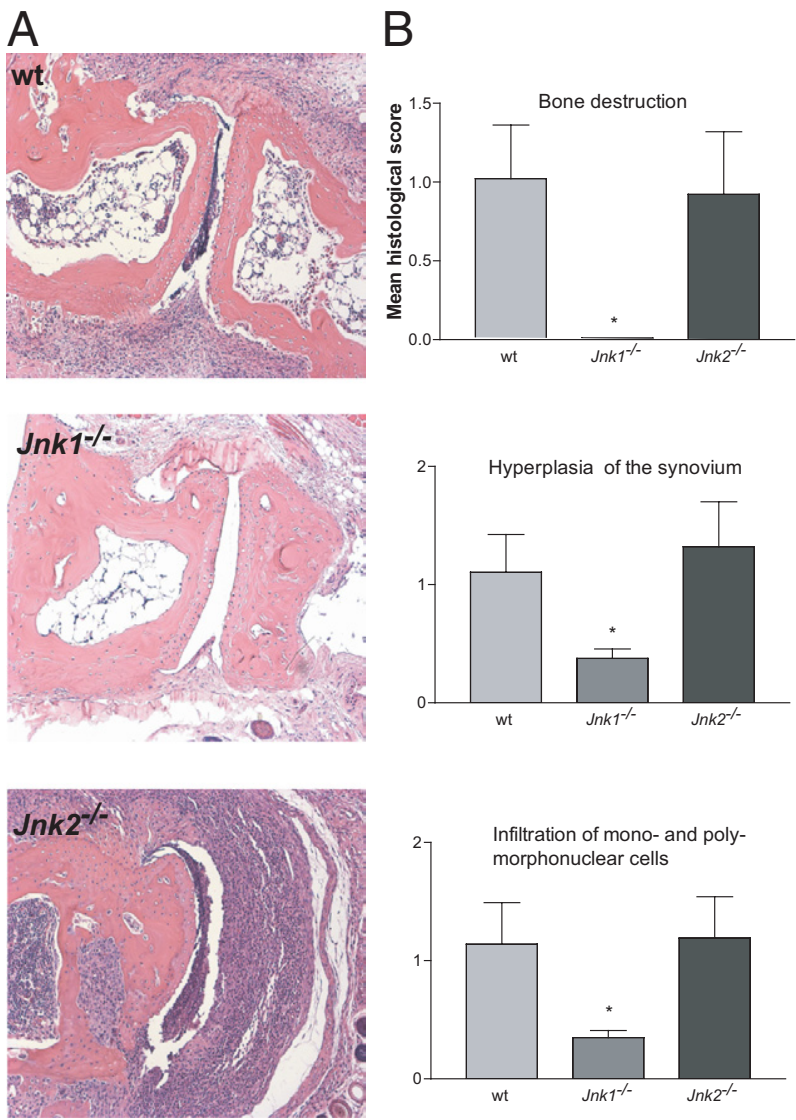

Figure 2. Histological analysis confirms the lack of inflammation in joint from $J n k 1^{-/-}$mice with CIA. A: Representative H\&E-stained sections from hind paws of wt, $J n k 1^{-/-}$, and $J n k 2^{-/-}$mice obtained at day 70 after immunization. There was absent or minimal inflammation in the $J n k 1^{-/}$ section. B: The severity of arthritis was histologically evaluated using the following parameters: bone destruction, hyperplasia of the synovium, and infiltration of monomorphonuclear and polymorphonuclear cells. Each parameter was scored as described in Materials and Methods. ${ }^{*} P<0.05$ $(n=10)$. 
A

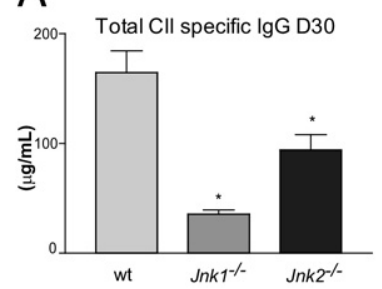

B

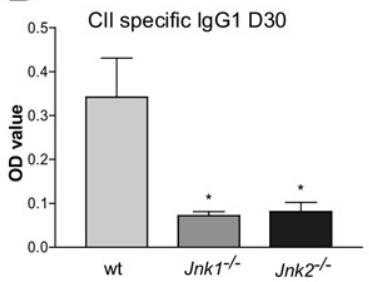

C

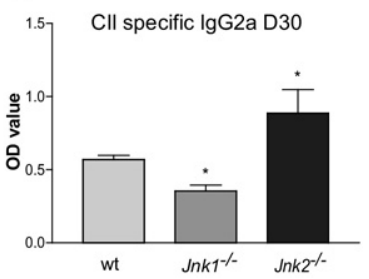

Figure 3. Altered humoral immunity in response to CII in $I n k 1^{-/-}$and $I n k 2^{-/-}$mice with CIA indicates a substantial Th1 response in the JNK2-deficient mice. A: Total CII-specific IgG serum levels in wt, $J n k 1^{-/-}$, and $J n k 2^{-/-}$mice on day 30 after immunization. IgG1 (B) and $\operatorname{IgG} 2 \mathrm{a}(\mathbf{C})$ levels in wt, $J n k 1^{-/-}$, and $J n k 2^{-/-}$ mice were measured on day 30 after immunization. ${ }^{*} P<0.05(n=10)$. gent roles of JNK1 and JNK2 observed during the progression of $\mathrm{CIA}$, we compared the expression levels of Cll-specific IgG between wt, Jnk1 $1^{-1-}$, and Jnk2 ${ }^{-1-}$ mice during the disease course. As expected, Jnk1 $1^{-1-}$ mice showed dramatically reduced levels of total IgG compared with wt mice at days 30 and 70 after the first immunization (Figure $3 \mathrm{~A}$ and data not shown, respectively). Surprisingly, the total IgG response in Jnk2 ${ }^{-1-}$ mice was also significantly reduced, although not to the same extent as observed in serum from $\mathrm{Jnk} 1^{-1-}$ mice, compared with that in wt mice at both time points studied.

To assess the IgG subclass composition of the $A b$ response, we measured the levels of the Cll-specific IgG1 and IgG2a isotypes in serum on day 30 after the first immunization. wt mice generated substantial Cll-specific $\operatorname{lgG} 1$ and $\lg$ G2a titers, and the ratio of $\lg$ G2a/lgG1 was 1.7 (Figure 3B). In contrast, the titers of IgG1 and IgG2a in serum from $\mathrm{Jnk} 1^{-1-}$ mice were marginal compared with wt serum with an IgG2a/lgG1 ratio of 5.0. Interestingly, although $J n k 2^{-I-}$ mice showed significantly reduced IgG1 titers compared with wt mice, the levels of CII-specific IgG2a were significantly increased, revealing an IgG2a/lgG1 ratio of 11.1 (Figure 3B), strongly suggesting a predominant type 1 helper T cell (Th1) immune response.

\section{Altered Cellular Immunity in JNK-Deficient Mice}

To investigate the importance of JNK1 and JNK2 for the generation of T-cell-mediated immunity, we induced DTH responses by immunizing the mice intradermally with $\mathrm{CII}$ in CFA, followed by intradermal challenge in the ear using CII without the adjuvant 12 days later. At 24 hours after challenge, wt and Jnk2 $2^{-1-}$ mice showed a strong response, with $50 \%$ and $75 \%$ ear swelling, respectively. In contrast, Jnk1 $1^{-1-}$ mice were almost unresponsive $(<10 \%$ ear swelling) (Figure 4A). Next, we wanted to investigate whether the lack of response in $\mathrm{Jnk} \mathrm{1}^{-1-}$ mice was directly related to a deficient T-cell response or if this might be due to an inability of myeloid cells (ie, macrophages and/or dendritic cells) to respond to microbial products present in CFA. We addressed this by challenging wt, Jnk1 $1^{-1-}$, and Jnk2 ${ }^{-1-}$ mice in an alternative model of DTH that is independent of CFA. Thus, we assessed cutaneous contact hypersensitivity in animals using DNFB, which generates a specific cutaneous T-cell-mediated allergic response on repeated allergen contact. ${ }^{20}$ Similar to what we observed using Cll, Jnk2 ${ }^{-1-}$ mice showed a strong response to DNFB 24 hours after challenge. However, although the response in JNK2-deficient mice was significantly increased compared with wt mice, there was no difference in ear swelling between wt and Jnk $1^{-1-}$ mice (Figure 4B). Thus, although there is an alteration in cellular immunity in Jnk $1^{-1-}$ mice when CFA is used for immunization, this is not the case in the absence of the adjuvant, suggesting that JNK1-deficient antigen-presenting cells might have an inadequate capability to respond to certain microbial agents.

In addition, because IL-17 produced by inflammatory Th17 may be involved in the pathogenesis of RA and in $\mathrm{CIA},{ }^{21}$ we wanted to explore the ability of JNK1- and JNK2-deficient T cells to differentiate into IL-17-producing effector cells. Thus, we purified naïve $\mathrm{CD} 4^{+} \mathrm{CD} 62 \mathrm{~L}^{+}$ $\mathrm{T}$ cells from wt, Jnk1 $1^{-1-}$, and Jnk2 ${ }^{-1-}$ mice and differentiated them in the presence of wt activated protein $\mathrm{C}$, anti-CD3, transforming growth factor- $\beta$, and IL-6. Five days after stimulation, the $T$ cells were restimulated with phorbol 12-myristate 13-acetate (PMA) and ionomycin and subjected to intracellular fluroscence-activated cell sorting (FACS) staining for the expression of IL-17. Although wt T cells produced significant amounts of IL-17 when restimulated, the amount of IL-17 produced from JNK1- and JNK2-deficient T cells was even higher (see Supplemental Figure S1 at $h$ ttp://ajp.amjpathol.org).

\section{Failure of Jnk1 ${ }^{-1-}$ Macrophages to Up-Regulate Costimulatory Molecules}

To investigate whether antigen presenting cells (APC) from Jnk $1^{-1-}$ mice had a distorted capability to provide efficient stimulation for cellular immunity to occur, we generated BMMs from wt, Jnk $1^{-1-}$, and Jnk $2^{-1-}$ mice and stimulated them with the bacterial cell wall protein
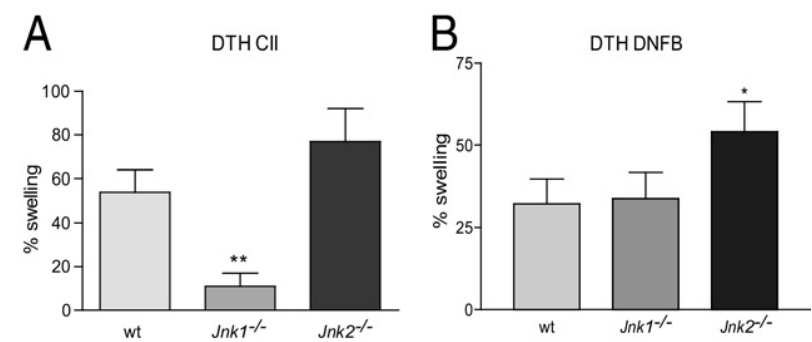

Figure 4. Altered cellular immunity in JNK-deficient mice. A: DTH response in wt, $J n k 1^{-1-}$, and $I n k 2^{-/-}$mice 24 hours after challenge with CII. B: The results at 24 hours after challenge with DNFB, suggesting a deficiency in the ability of $J n k 1^{-/-}$antigen-presenting cells in responding to certain microbial products. Antigen-induced ear swelling is presented as the percentage increase in ear thickness of the stimulated ear compared with the control ear. ${ }^{*} P<0.05,{ }^{* *} P<0.01$ ( $n=7$ for CII and $n=6$ for DNFB) 
A
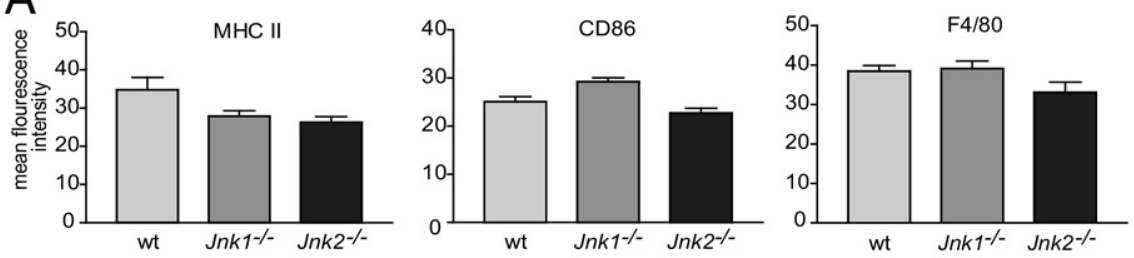

B
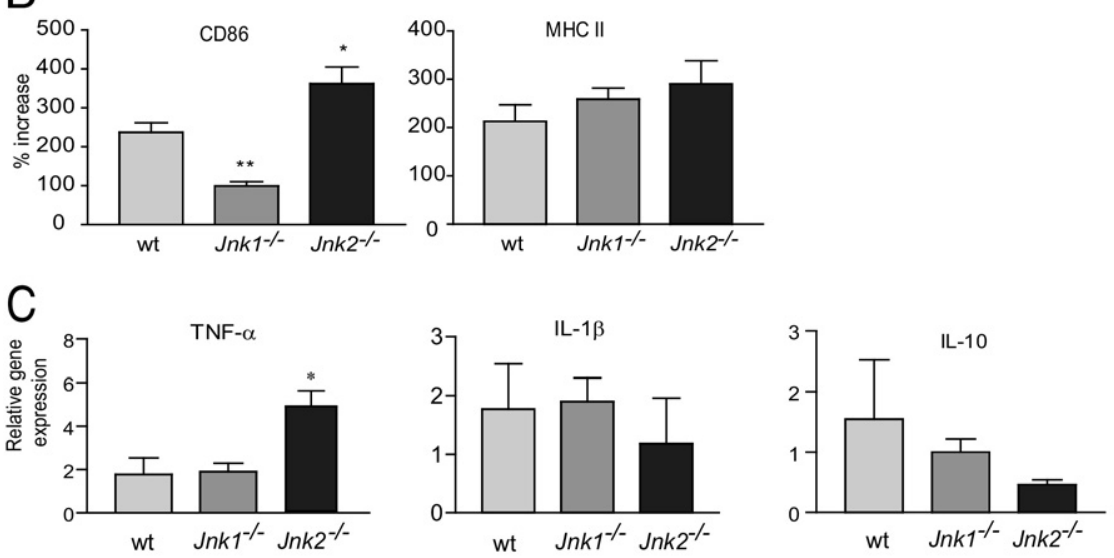

D

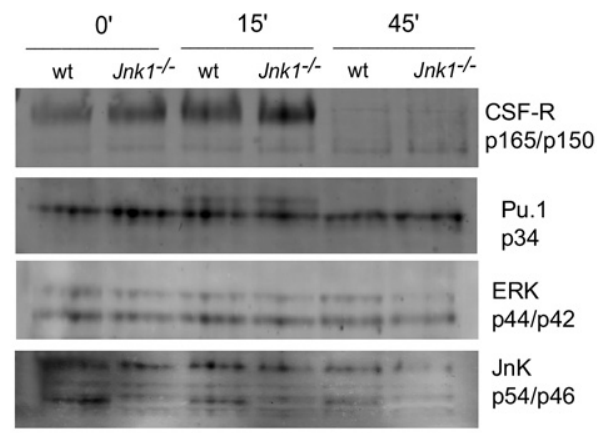

Figure 5. Failure of $I n k 1^{-/-}$macrophages to up-regulate costimulatory molecule CD86. A: FACS analysis of MHC II (I-E $\left.{ }^{\mathrm{k}}\right), \mathrm{CD} 86$, and F4/80 expression levels (mean fluorescence intensity) in unstimulated BMMs from wt, $J n k 1^{-/-}$, and $J n k 2^{-1-}$ mice $(n=6)$. B: Percentage increase in mean fluorescence intensity in BMMs stimulated with LPS $(10 \mathrm{ng} / \mathrm{mL})$ for 24 hours $(n=6)$. C: The BMMs were stimulated for 6 hours with LPS (10 $\mathrm{ng} / \mathrm{mL}$ ), and cytokine mRNA levels were measured by real-time PCR analysis. ${ }^{*} P<0.05,{ }^{* *} P<$ $0.001(n=6)$. D: Protein extracts were prepared from wt and $J n k 1^{-/-}$BMMs stimulated with LPS $(10 \mathrm{ng} / \mathrm{mL})$ for the indicated time points and analyzed by immunoblotting. Data suggest that JNK1 or JNK2 is sufficient for macrophage differentiation and maturation. ERK, extracellular signal-regulated kinase. E: RAW 264.7 cells were treated with SP600125 JNK inhibitor $(25 \mu \mathrm{mol} / \mathrm{L})$ for 1 hour before 24-hour LPS stimulation (1 $\mu \mathrm{g} / \mathrm{mL}$ ) and co-cultured with carboxyfluorescein diacetate succinimyl ester-marked primary murine $\mathrm{T}$ cells for 96 hours. Although the dividing $\mathrm{CD}^{+}$cells in the control go through at least three cell divisions, most $\mathrm{CD} 4^{+}$cells stimulated with SP600125-treated RAW cells remain quiescent $(76.8 \%$ versus $30.5 \%$ dividing cells). CFSE, carboxyfluorescein diacetate succinimyl ester.
LPS. Similar numbers of BMMs were generated from wt, Jnk $1^{-1-}$, and Jnk2 ${ }^{-1-}$ bone marrow, and no difference in the expression level (mean fluorescence intensity) of F4/ 80, MHC II, or CD80 was detected between the groups (Figure 5A). Moreover, JNK has phosphorylated the PU.1 transcription factor, which is critical for differentiation of macrophages and is required for the expression of the macrophage colony-stimulating factor receptor (CSF1R). ${ }^{22}$ However, we observed no difference in the expression levels of PU.1 or the CSF-1R in wt and Jnk $1^{-1-}$ BMMs (Figure 5D). We also did not detect differences in the expression levels of CSF-1R or the ability of JNK1deficient BMMs to phosphorylate PU.1 in response to LPS (Figure 5D). LPS stimulation has previously induced a rapid down-regulation of the CSF-1R in macrophages. ${ }^{23}$ Accordingly, LPS treatment of BMMs for 45 minutes induced a marked down-regulation of the CSF$1 \mathrm{R}$, and no difference was detected between wt and JNK1-deficient BMMs. Collectively, these data suggest that JNK1 or JNK2 is sufficient for macrophage differentiation and maturation.

When wt BMM was stimulated with LPS for 24 hours, the expression levels (mean fluorescence intensity) of
MHC II and CD86 increased by $212 \%$ and $237 \%$, respectively (Figure 5B). In Jnk2 ${ }^{-1-}$ BMMs, these numbers were even more pronounced (290\% and $362 \%$, respectively). In contrast, although Jnk1-1- BMMs were able to upregulate $\mathrm{MHC}$ II to similar levels as wt mice (258\%), a comparably modest increase in CD86 expression (99\%) was observed (Figure 5B). We also analyzed the expression of CD86 after stimulation of wt and JNK1-deficient BMMs with mycobacterium. Similar to what we observed using LPS, although not as pronounced, mycobacterium induced a significant up-regulation of CD86 in wt BMMs $(117 \%)$, whereas the increase in expression was modest in JNK1-deficient macrophages (43\%) (data not shown). These results suggest that JNK1-deficient macrophages have an inferior costimulatory capacity compared with wt and Jnk2 $2^{-1-}$ macrophages and might be of particular importance because CD86 has been the prominent costimulatory molecule required for efficient activation of naïve CD4 ${ }^{+}$T cells in vivo. ${ }^{24}$ To validate these results, we cultured a murine macrophage cell line (ie, RAW 264.7 cells) and pretreated the cells with the specific JNK inhibitor SP600125 for 1 hour before stimulation for 24 hours with $1 \mu \mathrm{g} / \mathrm{mL}$ LPS. Hereafter, the cells were stained 
with CD86 Abs and run on a flow cytometer. A dosedependent down-regulation of CD86 was shown (see Supplemental Figure S2 at http://ajp.amjpathol.org). Next, we made a co-culture of RAW cells and primary T cells to determine whether SP600125-treated RAW cells were able to activate primary naîve $\mathrm{CD} 4^{+} \mathrm{T}$ cells. The results are shown in Figure 5E. As judged by the percentage of dividing cells, the SP600125-pretreated RAW cells failed to induce significant activation of $\mathrm{CD} 4^{+} \mathrm{T}$ cells compared with control-treated RAW cells $30.5 \%$ versus $76.8 \%$ dividing cells). Furthermore, although the $\mathrm{CD}^{+}{ }^{+}$cells activated by control-treated RAW cells went through at least three cell divisions, only a few of the $\mathrm{CD} 4^{+}$cells cultured with SP600125-treated RAW cells completed one cell cycle; most of them never divided.

Next, we evaluated the ability of BMMs from wt, Jnk $1^{-1-}$, and Jnk2 $2^{-I-}$ mice to produce cytokines in response to LPS. Although the lack of JNK1 did not seem to affect the production of any of the cytokines investigated (ie, TNF- $\alpha$, IL-1, IL-10, and IL-6; Figure 5C and data not shown), lack of JNK2 resulted in a significant increase in TNF- $\alpha$ production. Thus, although JNK1 deficiency appears to have a negative effect on the costimulatory capacity in macrophages, lack of JNK2 provides more efficient costimulation and increases the production of the pro-inflammatory cytokine TNF- $\alpha$ in response to LPS.

\section{Divergent Roles of JNK1 and JNK2 in the Development of Serum-Induced Arthritis}

To determine the role of the JNK signaling pathway in the effector phase of arthritis, we used the serum transfer model of inflammatory arthritis $(\mathrm{K} / \mathrm{B} \times \mathrm{N})$. This model is independent of both $\mathrm{B}$ and $\mathrm{T}$ cells and does not require adjuvants for the development of arthritis. ${ }^{12}$ After serum transfer, wt and Jnk2 $2^{-I-}$ mice displayed marked physical inflammation, as judged by clinical score and ankle swelling. The development of inflammatory arthritis in JNK2deficient animals was significantly more rapid and severe compared with the wt control $(P<0.001$ at days 5 through 9 , as judged by $\delta$ ankle thickness, and $P<$ 0.05 at days 3 through 8 , as judged by clinical score; Figure 6A).

In contrast, Jnk1-1- mice developed weak arthritis compared with wt animals $(P<0.05$ at days 10 through 22 , as judged by $\delta$ ankle thickness, and $P<0.005$ at days 10 through 20, as judged by clinical score). Although we could not detect a deficiency in the development and maturation of BMMs from Jnk1 $1^{-1-}$ mice, their inability to up-regulate CD86 in response to LPS suggests that JNK1-deficient macrophages are dysfunctional, at least in some aspects.

To further investigate whether the modest inflammatory response observed in JNK1-deficient mice could be due to inefficient macrophage function, we adoptively transferred wt BMMs to Jnk $1^{-1-}$ mice before the administration of $\mathrm{K} / \mathrm{BxN}$ serum. This approach resulted in a significant increase in clinical score $(P<0.05$ at days 5,9 , and 11; Figure 6B). An increase in ankle thickness was also
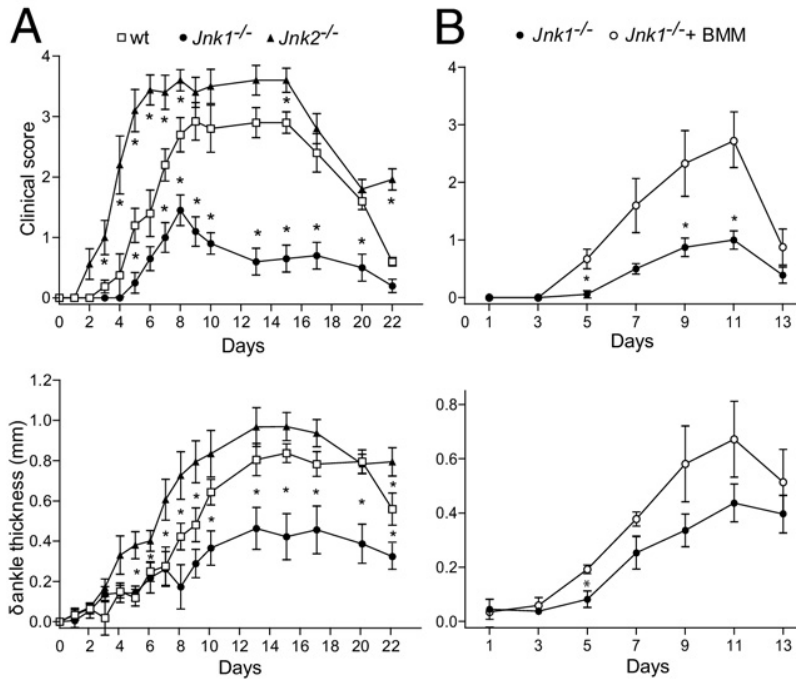

Figure 6. Divergent roles of JNK1 and JNK2 in the development of seruminduced arthritis. Clinical arthritis in wt, $J n k 1^{-/-}$, and $J n k 2^{-/-}$mice after injection of $\mathrm{K} / \mathrm{BxN}$ serum i.p., as described in Materials and Methods. A: The mice were assessed for arthritis development by clinical index score and ankle thickness. B: wt BMMs were grown in culture, as described in Materials and Methods, and i.v. injected via tail vein into $\mathrm{Ink1^{-/- }}$ mice 1 day before and 1 and 3 days after serum transfer. The values represent the mean $\pm S E M$ ${ }^{*} P<0.05$ compared with $\mathbf{A}$ (wt mice) or $\mathbf{B}$ (mice not receiving BMMs under parallel conditions).

observed, although this was only significant at day 5 after serum transfer (Figure 6B).

\section{Discussion}

In this study, we have shown distinct roles for JNK1 and JNK2 in two separate mouse models of RA. Although JNK1 is critically required and able to substitute for lack of JNK2 in the initiation and effector phase of inflammatory arthritis and even exacerbate clinical signs of arthritis, JNK2 does not possess these abilities. An important function for JNK during inflammatory arthritis appears to be control of innate immunity through macrophage activation and cytokine production.

The role of the JNK kinases, in inflammatory arthritis using JNK2-deficient mice and JNK-specific inhibitors (ie, SP600125, recognizing both JNK1 and JNK2), has been previously addressed. ${ }^{3,4}$ Blocking JNK1 and JNK2 using SP600125 in a rat model of adjuvant-mediated arthritis had a notable effect on inhibition of radiographical damage through inhibition of matrix-destroying metalloproteinases. This was confirmed in JNK1- and JNK2deficient mouse synovial cell lines, although JNK2 seemed to be the relatively more important isotype for matrix-destroying metalloproteinase production. In contrast, chemical inhibition of both JNK1 and JNK2 in this model had a modest effect on the inflammatory response, as judged by paw swelling. ${ }^{3}$ In an additional study, ${ }^{4}$ using JNK2 knockout mice and a mouse model for passive CIA, the same group showed enhanced clinical arthritis in JNK2-deficient mice compared with the wt control, although synovial inflammation did not differ and cartilage and bone destruction levels were slightly better 
in JNK2-deficient mice compared with wt mice. In contrast, in a study ${ }^{5}$ of TNF- $\alpha$-mediated joint disease, knockout of Jnk1 did not change the disease course, as judged by clinical and histological features. Recently, Guma and coworkers $^{6,7}$ showed reduced disease severity in Jnk $1^{-1-}$ mice in two separate models of AIA when comparing mice deficient in JNK1 and JNK2 with wt animals.

We show that JNK1-deficient mice are almost completely protected from CIA, whereas JNK2-deficient mice developed more severe clinical signs of arthritis, with a higher incidence compared with wt mice. This is opposite of the results reported for the Jnk1-l- hTNFtg mouse; however, the TNF- $\alpha$ overexpression model does not involve the autoimmune disease phase and this may explain the differing results in the models. ${ }^{5}$ Thus, it seems likely that, although inhibition of both JNK1 and JNK2 is required for inhibition of matrix-destroying metalloproteinases from synovial cells during the effector phase of arthritis, the two isotypes have different roles in other aspects of the inflammatory response: JNK1 and JNK2 isoforms may compete for substrates and, although JNK1 is able to compensate for lack of JNK2 by up-regulating its activity, JNK2 is not able to do the same, producing apparent opposing phenotypes of the JNK1- and JNK2deficient mice. ${ }^{25}$ The study ${ }^{5}$ of JNK1-deficient hTNFtg mice is in favor of this theory, in which the number of cells expressing phosphorylated JNK was significantly reduced in Jnk1-/- hTNFtg mice compared with Jnk $1^{+/+}$ hTNFtg mice. However, a difference in phosphorylation of cJun within the synovial membrane could not be detected. Likewise, JNK2 was equally expressed in the synovial tissue, indicating that JNK2 was able to phosphorylate cJun in vivo. Indeed, in vitro studies of Jaeschke et $\mathrm{al}^{25}$ did show that JNK2 is able to phosphorylate cJun; however, its function is redundant compared with JNK1. Thus, lack of JNK1 may result in attenuation of inflammatory disease because of a reduced ability of JNK2 to efficiently phosphorylate its substrates compared with JNK1. Alternatively, JNK1 and JNK2 may have opposing effects under certain conditions.

JNK has previously been involved in T-cell activation and differentiation. ${ }^{13,14,26}$ Although JNK2 was required for sufficient interferon- $\gamma$ production to induce Th1 differentiation, ${ }^{14,26}$ inhibition of JNK1 results in preferential differentiation to Th2 cells, through enhanced production of Th2 cytokines, leading to an inability to heal skin lesions on Leshmania infection. ${ }^{27}$

In CIA, mycobacterium adjuvant (ie, CFA) induces a strong immune response, leading to a cytokine environment favoring antigen-specific Th1 differentiation. ${ }^{28}$ This suggested that JNK1 deficiency, through enhanced Th2 cytokine production, might offer protection against CIA. In our studies, JNK1-deficient mice were protected from CIA. However, the minimal Cll-specific IgG levels suggested that no functional T-cell response is generated in Jnk $1^{-1-}$ mice. Surprisingly, the total Cll-specific IgG levels in Jnk2 ${ }^{-1-}$ mice were also lower compared with wt mice, although not to the same extent as in Jnk $1^{-1-}$ mice. More important, the $\mathrm{lgG} 2 \mathrm{a} / \mathrm{lgG} 1$ ratio in serum from Jnk2 $2^{-1-}$ mice was sevenfold higher compared with what we observed in wt mice and the level of IgG2a alone was significantly higher than in wt mice (Figure 3B). In the Jnk $1^{-1-}$ mice, the IgG2a/lgG1 ratio in serum was threefold higher than in wt mice, but the level of IgG2a alone was significantly reduced compared with Jnk2 ${ }^{-1-}$ and wt mice. This indicates a relative dominance of Th1 cytokines compared with wt mice in both $J n k 1^{-1-}$ and Jnk2 $2^{-1-}$ mice, which is opposite to what other studies ${ }^{13,14}$ have shown. However, because mycobacterium in CFA has induced a Th1 response, ${ }^{11}$ it seems possible that, under the existing experimental conditions, the $T$ cells were able to differentiate into Th1 cells in the Jnk2 ${ }^{-1-}$ and Jnk1 $1^{-1-}$ mice, although this was less pronounced for $\mathrm{Jnk} \mathrm{1}^{-1-}$ mice, in agreement with the substantial Th2 response seen in other studies. ${ }^{13}$ Furthermore, because the severity of CIA is positively correlated with the $\operatorname{lgG}$ autoantibody response to $\mathrm{Cll}$ in general ${ }^{29}$ and the predominance of autoreactive IgG2a Abs in particular, ${ }^{30}$ this could, at least partly, explain the resistance to CIA in $\mathrm{Jnk}^{-1-}$ mice and the enhanced severity of arthritis observed in $J n k 2^{-1-}$ mice compared with wt mice. Furthermore, we show that Jnk $1^{-1-}$ mice are able to generate a functional T-cell-mediated immune response against DNFB in a model of cutaneous contact hypersensitivity that is independent of an adjuvant (ie, CFA) and that JNK1-deficient T cells are able to differentiate into IL-17-producing effector cells. These data are in agreement with observations of comparable T-cell proliferation in $\mathrm{Jnk}^{-1-}$ and $\mathrm{Jnk2^{-1- }}$ mice in the AlA model ${ }^{7}$ and collectively suggest that the protective effect of JNK1 inhibition is dependent on an inability of $\mathrm{Jnk1^{-/- }}$ antigenpresenting cells to respond to certain microbial agents, rather than insufficiency of cell-mediated immunity in Jnk $1^{-1-}$ mice. A recent study ${ }^{31}$ has proposed that JNK1 activity is induced in myeloid cells in response to microbial pathogens and shows that $J n k 1^{-1-}$ mice are almost completely protected from the induction of experimental autoimmune encephalomyelitis. In addition, the migration of macrophages was impaired in the AIA model. ${ }^{7}$ Macrophages are required for $\mathrm{CIA}$ and have been the primary antigen-presenting cells in the immune response toward type II collagen. ${ }^{32-34}$

Both JNK1 and JNK2 are constitutively expressed in macrophages, and a recent study, ${ }^{35}$ using chemical inhibition of all JNK isoforms, has suggested that JNK signaling is required for differentiation of the monocyte/ macrophage lineage, expression of the CSF-1R, and phosphorylation of the PU.1 transcription factor, which is important for development of the monocyte/macrophage lineage. ${ }^{22,36,37}$ We show that JNK1 (or JNK2) is sufficient for differentiation of the monocyte/macrophage lineage, expression of the CSF-1R, and phosphorylation of the PU.1 transcription factor. However, JNK1-deficient macrophages challenged with microbial pathogens (eg, LPS or mycobacterium) demonstrate dramatically reduced CD86 expression compared with wt macrophages. In line with this result, the murine macrophage cell line RAW 264.7 showed dose-dependent down-regulation of CD86 and could not stimulate primary murine naïve $\mathrm{CD}^{+} \mathrm{T}$ cells to divide when treated with a JNK inhibitor. In contrast, JNK2-deficient macrophages demonstrated a significant increase in CD86 expression compared with wt. 
Thus, JNK1 is important for adjuvant induced up-regulation of B7 costimulation on macrophages, whereas the role for JNK2 is inferior or possibly converse. One previous study ${ }^{38}$ has addressed the relative importance of the JNK isoforms for expression of B7 on macrophages in response to LPS. However, in this study, inhibition of JNK1 or JNK2 by the respective small-interfering RNA had a similar, but weak, inhibitory effect on expression of CD80, which possibly can be explained by lack of complete inhibition.

LPS acts primarily on toll-like receptor 4 on macrophages to activate $\mathrm{JNK}^{39-41}$ and JNK has, in addition to regulation of costimulation, been involved in LPS-mediated expression of chemokines and cytokines (ie, TNF- $\alpha$ and IL-1) in macrophages. ${ }^{42,43}$ In our study, JNK1-deficient and wt BMMs produced similar levels of the proinflammatory cytokines, TNF- $\alpha$ and IL-1, in response to LPS, whereas JNK2-deficient BMMs produced markedly elevated levels of TNF- $\alpha$ and lower levels of IL-10 compared with wt BMMs. These results correlate with a previous study demonstrating similar levels of IL-1 and IL-10 in wt and JNK1-deficient BMMs after LPS stimulation ${ }^{31}$ and similar levels of IL-1 $\beta$ after TNF- $\alpha$ stimulation. ${ }^{7}$ We propose that, in an adjuvant and T-cell-dependent model of inflammation, JNK1 is a potent activator of innate immunity by triggering up-regulation of costimulation required for initiation of an adaptive T-cell response. In contrast, JNK2 activity alone most likely cannot induce satisfactory expression of costimulatory molecules and production of pro-inflammatory cytokines to induce arthritis in this model.

Han et $\mathrm{al}^{4}$ show enhanced clinical arthritis in JNK2deficient mice compared with the wt control using a Tcell-independent mouse model for passive CIA. Interestingly, LPS was used in that study to exacerbate the inflammatory response. ${ }^{4}$ Our results, showing enhanced TNF- $\alpha$ production from JNK2-deficient macrophages in response to LPS, can partly explain the outcome in that study.

To compare the role of JNK1 with JNK2 in an arthritis model that is independent of the autoimmune induction phase, we chose the $\mathrm{K} / \mathrm{BxN}$ model of serum-induced arthritis. This model is T-cell independent ${ }^{44,45}$ but dependent on immune complexes, complement, ${ }^{44}$ neutrophils, mast cells, ${ }^{46,47}$ macrophages, ${ }^{48}$ and the production of pro-inflammatory cytokines (ie, IL-1 $\beta$ and TNF- $\alpha$ ) for progression. ${ }^{49}$ Surprisingly, although this model is independent of an adjuvant, such as mycobacterium or LPS, we observed a significant protection in JNK1-deficient mice, whereas an exacerbated response was detected in JNK2-deficient mice. Although the precise mechanistic explanation for the divergent roles of JNK1 and JNK2 during serum-induced arthritis remains to be investigated, the protective role of JNK1 deficiency is macrophage dependent, because adoptive transfer of wt BMMs to the JNK1-deficient mice restored their susceptibility to serum-induced arthritis. This supplements the results shown by Guma and coworkers, ${ }^{6}$ who also saw a protection in Jnk $1^{-1-}$ mice, in this case suspected to depend on diminished mast cell degranulation and cytokine production, collectively suggesting a dual effect on innate immunity during the inflammatory response in the model.

In conclusion, our results demonstrate divergent abilities for the JNK kinases in two distinct models of arthritis and suggest that the least common denominator determining the outcome of JNK1 and JNK2 deficiency during the autoimmune initiation phase, and during the effector phase of inflammatory arthritis, is related to macrophage function. Finally, the previously suggested approach of achieving major blockade of JNK signaling in inflammatory arthritis through blockade of both JNK1 and JNK2 ${ }^{4,5}$ should be revised, because isoform-specific inhibition of JNK1 likely will be a more efficient way of inhibiting the inflammatory response.

\section{Acknowledgment}

The KRN mice were provided by Diane Mathis and Christophe Benoist (IGMBC, Illkirk, France).

\section{References}

1. van den Berg WB: Anti-cytokine therapy in chronic destructive arthritis. Arthritis Res 2001, 3:18-26

2. Schett G, Tohidast-Akrad M, Smolen JS, Schmid BJ, Steiner CW, Bitzan P, Zenz P, Redlich K, Xu Q, Steiner G: Activation, differential localization, and regulation of the stress-activated protein kinases, extracellular signal-regulated kinase, c-JUN N-terminal kinase, and p38 mitogen-activated protein kinase, in synovial tissue and cells in rheumatoid arthritis. Arthritis Rheum 2000, 43:2501-2512

3. Han Z, Boyle DL, Chang L, Bennett B, Karin M, Yang L, Manning AM Firestein GS: c-Jun N-terminal kinase is required for metalloproteinase expression and joint destruction in inflammatory arthritis. J Clin Invest 2001, 108:73-81

4. Han Z, Chang L, Yamanishi Y, Karin M, Firestein GS: Joint damage and inflammation in C-Jun $\mathrm{N}$-terminal kinase 2 knockout mice with passive murine collagen-induced arthritis. Arthritis Rheum 2002, 46: $818-823$

5. Koller M, Hayer S, Redlich K, Ricci R, David JP, Steiner G, Smolen JS, Wagner EF, Schett G: JNK1 is not essential for TNF-mediated joint disease. Arthritis Res Ther 2005, 7:R166-R173

6. Guma M, Kashiwakura J, Crain B, Kawakami Y, Beutler B, Firestein GS, Kawakami T, Karin M, Corr M: JNK1 controls mast cell degranulation and IL-1 \{beta\} production in inflammatory arthritis. Proc Natl Acad Sci U S A 2010, 107:22122-22127

7. Guma M, Ronacher LM, Firestein GS, Karin M, Corr M: JNK-1 deficiency limits macrophage-mediated antigen-induced arthritis. Arthritis Rheum 2011, 63:1603-1612

8. Williams RO: Collagen-induced arthritis in mice. Methods Mol Med 2007, 136:191-199

9. Joe B, Griffiths MM, Remmers EF, Wilder RL: Animal models of rheumatoid arthritis and related inflammation. Curr Rheumatol Rep 1999, 1:139-148

10. Campbell IK, Hamilton JA, Wicks IP: Collagen-induced arthritis in C57BL/6 ( $\mathrm{H}-2 \mathrm{~b})$ mice: new insights into an important disease model of rheumatoid arthritis. Eur J Immunol 2000, 30:1568-1575

11. Inglis JJ, Criado G, Medghalchi M, Andrews M, Sandison A, Feldmann M, Williams RO: Collagen-induced arthritis in C57BL/6 mice is associated with a robust and sustained T-cell response to type II collagen. Arthritis Res Ther 2007, 9:R113

12. Monach PA, Benoist C, Mathis D: The role of antibodies in mouse models of rheumatoid arthritis, and relevance to human disease. Adv Immunol 2004, 82:217-248

13. Dong C, Yang DD, Wysk M, Whitmarsh AJ, Davis RJ, Flavell RA: Defective T cell differentiation in the absence of Jnk1. Science 1998 , 282:2092-2095 
14. Yang DD, Conze D, Whitmarsh AJ, Barrett T, Davis RJ, Rincon M, Flavell RA: Differentiation of CD4+ T cells to Th1 cells requires MAP kinase JNK2. Immunity 1998, 9:575-585

15. Kouskoff V, Korganow AS, Duchatelle V, Degott C, Benoist C, Mathis D: Organ-specific disease provoked by systemic autoimmunity. Cell 1996, 87:811-822

16. Schett G, Tuerk B: Bone histomorphometry in arthritis models 1 Methods Mol Med 2007, 135:269-283

17. Monach PA, Mathis D, Benoist C: The K/BxN arthritis model. Curr Protoc Immunol 2008, Chapter 15:Unit 15.22

18. Bonnesen B, Orskov C, Rasmussen S, Holst PJ, Christensen JP, Eriksen KW, Qvortrup K, Odum N, Labuda T: MEK kinase 1 activity is required for definitive erythropoiesis in the mouse fetal liver. Blood 2005, 106:3396-3404

19. Seki N, Sudo Y, Yoshioka T, Sugihara S, Fujitsu T, Sakuma S, Ogawa T, Hamaoka T, Senoh H, Fujiwara H: Type II collagen-induced murine arthritis, I: induction and perpetuation of arthritis require synergy between humoral and cell-mediated immunity. J Immunol 1988, 140: 1477-1484

20. Wang B, Feliciani C, Freed I, Cai Q, Sauder DN: Insights into molecular mechanisms of contact hypersensitivity gained from gene knockout studies. J Leukoc Biol 2001, 70:185-191

21. Nakae S, Nambu A, Sudo K, Iwakura Y: Suppression of immune induction of collagen-induced arthritis in IL-17-deficient mice. J Immunol 2003, 171:6173-6177

22. Zhang DE, Hetherington CJ, Chen HM, Tenen DG: The macrophage transcription factor PU.1 directs tissue-specific expression of the macrophage colony-stimulating factor receptor. Mol Cell Biol 1994, 14:373-381

23. Schroder K, Lichtinger M, Irvine KM, Brion K, Trieu A, Ross IL, Ravasi T, Stacey KJ, Rehli M, Hume DA, Sweet MJ: PU.1 and ICSBP control constitutive and IFN-gamma-regulated TIr9 gene expression in mouse macrophages. J Leukoc Biol 2007, 81:1577-1590

24. Lang TJ, Nguyen P, Peach R, Gause WC, Via CS: In vivo CD86 blockade inhibits CD4+ T cell activation, whereas CD80 blockade potentiates CD8+ T cell activation and CTL effector function. J Immunol 2002, 168:3786-3792

25. Jaeschke A, Karasarides M, Ventura JJ, Ehrhardt A, Zhang C, Flavell RA, Shokat KM, Davis RJ: JNK2 is a positive regulator of the cJun transcription factor. Mol Cell 2006, 23:899-911

26. Sabapathy K, Hu Y, Kallunki T, Schreiber M, David JP, Jochum W, Wagner EF, Karin M: JNK2 is required for efficient T-cell activation and apoptosis but not for normal lymphocyte development. Curr Biol 1999, 9:116-125

27. Constant SL, Dong C, Yang DD, Wysk M, Davis RJ, Flavell RA: JNK1 is required for $\mathrm{T}$ cell-mediated immunity against Leishmania major infection. J Immunol 2000, 165:2671-2676

28. Stasiuk LM, Abehsira-Amar O, Fournier C: Collagen-induced arthritis in DBA/1 mice: cytokine gene activation following immunization with type II collagen. Cell Immunol 1996, 173:269-275

29. Williams PJ, Jones RH, Rademacher TW: Correlation between IgG anti-type II collagen levels and arthritic severity in murine arthritis. Autoimmunity 1998, 27:201-207

30. Watson WC, Townes AS: Genetic susceptibility to murine collagen II autoimmune arthritis: proposed relationship to the IgG2 autoantibody subclass response, complement C5, major histocompatibility complex (MHC) and non-MHC loci. J Exp Med 1985, 162:1878-189

31. Tran EH, Azuma YT, Chen M, Weston C, Davis RJ, Flavell RA: Inactivation of JNK1 enhances innate IL-10 production and dampens autoimmune inflammation in the brain. Proc Natl Acad Sci U S A 2006 103:13451-13456

32. Campbell IK, Rich MJ, Bischof RJ, Hamilton JA: The colony-stimulating factors and collagen-induced arthritis: exacerbation of disease by M-CSF and G-CSF and requirement for endogenous M-CSF. J Leukoc Biol 2000, 68:144-150

33. Guery L, Chiocchia G, Batteux F, Boissier MC, Fournier C: Collagen II-pulsed antigen-presenting cells genetically modified to secrete IL-4 down-regulate collagen-induced arthritis. Gene Ther 2001, 8:18551862

34. Michaelsson E, Holmdahl M, Engstrom A, Burkhardt H, Scheynius A, Holmdahl R: Macrophages, but not dendritic cells, present collagen to T cells. Eur J Immunol 1995, 25:2234-2241

35. Himes SR, Sester DP, Ravasi T, Cronau SL, Sasmono T, Hume DA: The JNK are important for development and survival of macrophages. $\mathrm{J}$ Immunol 2006, 176:2219-2228

36. Mao C, Ray-Gallet D, Tavitian A, Moreau-Gachelin F: Differential phosphorylations of Spi-B and Spi-1 transcription factors. Oncogene 1996, 12:863-873

37. Valledor AF, Borras FE, Cullell-Young M, Celada A: Transcription factors that regulate monocyte/macrophage differentiation. J Leukoc Biol 1998, 63:405-417

38. Lim W, Gee K, Mishra S, Kumar A: Regulation of B7.1 costimulatory molecule is mediated by the IFN regulatory factor-7 through the activation of JNK in lipopolysaccharide-stimulated human monocytic cells. J Immunol 2005, 175:5690-5700

39. Poltorak A, He X, Smirnova I, Liu MY, Van HC, Du X, Birdwell D, Alejos E, Silva M, Galanos C, Freudenberg M, Ricciardi-Castagnoli P, Layton B, Beutler B: Defective LPS signaling in $\mathrm{C} 3 \mathrm{H} / \mathrm{HeJ}$ and $\mathrm{C} 57 \mathrm{BL} /$ 10ScCr mice: mutations in Tlr4 gene. Science 1998, 282:2085-2088

40. Muzio M, Natoli G, Saccani S, Levrero M, Mantovani A: The human toll signaling pathway: divergence of nuclear factor kappaB and JNK/ SAPK activation upstream of tumor necrosis factor receptor-associated factor 6 (TRAF6). J Exp Med 1998, 187:2097-2101

41. Qureshi ST, Lariviere L, Leveque G, Clermont S, Moore KJ, Gros P, Malo D: Endotoxin-tolerant mice have mutations in Toll-like receptor 4 (Tlr4). J Exp Med 1999, 189:615-625

42. Beutler B, Cerami A: Tumor necrosis, cachexia, shock, and inflammation: a common mediator. Annu Rev Biochem 1988, 57:505-518

43. Dinarello CA: Interleukin-1 and interleukin-1 antagonism. Blood 1991 , $77: 1627-1652$

44. Ji H, Ohmura K, Mahmood U, Lee DM, Hofhuis FM, Boackle SA, Takahashi K, Holers VM, Walport M, Gerard C, Ezekowitz A, Carrol MC, Brenner M, Weissleder R, Verbeek JS, Duchatelle V, Degott C, Benoist C, Mathis D: Arthritis critically dependent on innate immune system players. Immunity 2002, 16:157-168

45. Choe JY, Crain B, Wu SR, Corr M: Interleukin 1 receptor dependence of serum transferred arthritis can be circumvented by toll-like receptor 4 signaling. J Exp Med 2003, 197:537-542

46. Lee DM, Friend DS, Gurish MF, Benoist C, Mathis D, Brenner MB: Mast cells: a cellular link between autoantibodies and inflammatory arthritis. Science 2002, 297:1689-1692

47. Wipke BT, Allen PM: Essential role of neutrophils in the initiation and progression of a murine model of rheumatoid arthritis. J Immuno 2001, 167:1601-1608

48. Solomon S, Rajasekaran N, Jeisy-Walder E, Snapper SB, Illges H: A crucial role for macrophages in the pathology of $K / B \times N$ seruminduced arthritis. Eur J Immunol 2005, 35:3064-3073

49. Ditzel HJ: The K/BxN mouse: a model of human inflammatory arthritis. Trends Mol Med 2004, 10:40-45 